\title{
Empathie avec la matière
}

comment repenser la nature de l'action technique

Empathy with the matter

\section{Susanne Küchler et Graeme Were}

Traducteur : Ludovic Coupaye

\section{(2) OpenEdition}

Journals

Édition électronique

URL : https://journals.openedition.org/tc/4942

DOI : $10.4000 /$ tc. 4942

ISSN : 1952-420X

Éditeur

Éditions de l'EHESS

Édition imprimée

Date de publication : 1 décembre 2009

Pagination : 190-211

ISBN : 978-2-7351-1301-9

ISSN : 0248-6016

\section{Référence électronique}

Susanne Küchler et Graeme Were, « Empathie avec la matière », Techniques \& Culture [En ligne], 52-53 | 2009, mis en ligne le 01 août 2012, consulté le 29 septembre 2022. URL : http://

journals.openedition.org/tc/4942; DOI : https://doi.org/10.4000/tc.4942 


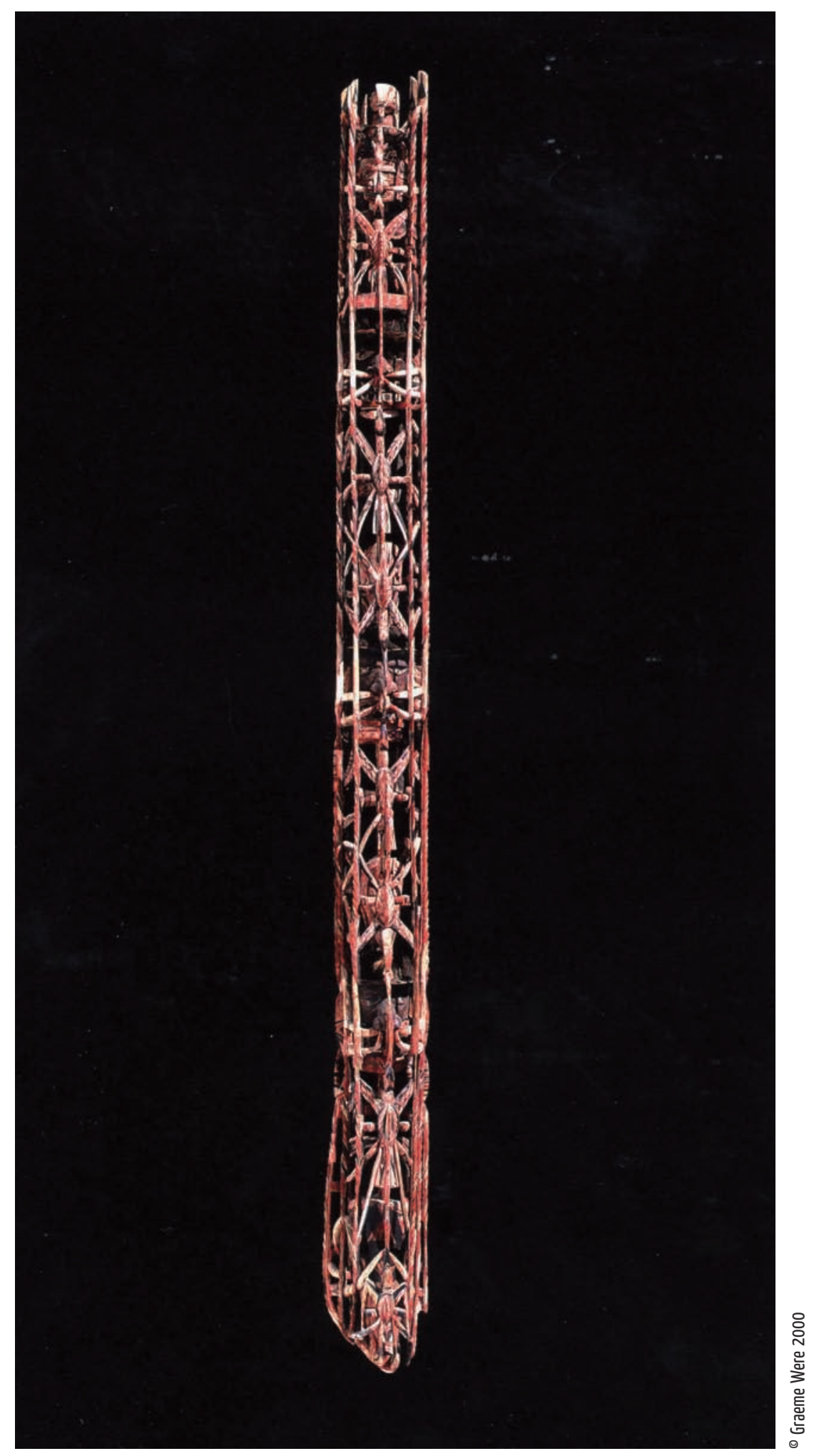




\title{
EMPATHIE AVEC LA MATIÈRE
}

\section{Comment repenser la nature de l'action technique}

\author{
En recourant à l'idée paradoxale « d'empathie avec la matière », Küchler et \\ Were mettent en lumière le rôle fondamental du concret dans l'imagination \\ sociale, via les techniques et la cognition.
}

La plupart des définitions de la technique font la distinction entre les éléments humains et non-humains. Le « non-humain » est généralement associé au contenu matériel, intrinsèque, technique, alors que l'» humain » tend à faire référence aux éléments circonstanciels du contexte (les facteurs sociaux et environnementaux), qui jouent alors le rôle de contraintes dans la transformation de la matière par les moyens techniques. Cette distinction a été contestée par les anthropologues français qui soutiennent que les processus techniques sont enchâssés dans un ensemble plus large de représentations culturelles (Lemonnier 1993; Sigaut 2002), ainsi que par les sociologues qui ont souligné l'importance de la relation analogique entre les deux systèmes, arguant que tous deux avaient la structure des réseaux d'information (Law 1992). D'autre part, Bruno Latour (1996) et Marilyn Strathern (1991) se sont distingués en envisageant le rôle des matériaux dans la formation de "connexions partielles » entre les systèmes sociaux et techniques. Alors que pour Latour les matériaux sont essentiels dans le maintien des relations entre le social et le technique, Strathern les considère dans leur analogie avec les substances corporelles 
qui sont déjà conçues comme relationnelles. Ces deux approches - bien que reconnaissant une relation entre le technique et le social - situent le cœur de l'action hors de la matière.

En gardant leurs contributions à l'esprit nous questionnerons, ici, la minimisation du rôle des matériaux dans la critique de la division entre les systèmes sociaux et techniques. Nous montrerons que les capacités transformatives des matériaux jouent un rôle fondamental dans leur sélection et leur utilisation et que, par ailleurs, toute transformation suppose des actions calculées qui sont, par nature, relationnelles. Nous affirmons que la sélection et la manipulation technique des matériaux vont de pair avec une empathie, une sorte d'intersubjectivité, qui permet la reconnaissance du potentiel de certains matériaux comme étant capables de répondre à des attentes essentielles et permettant de parvenir à des résultats spécifiques. En mettant au premier plan cette notion d'empathie avec les matériaux, nous croyons que notre article peut mener à une meilleure compréhension des relations sociales en Océanie.

La connaissance du rôle des propriétés physiques de la matière, non seulement au niveau des processus techniques et sociaux, mais aussi au niveau plus abstrait et général des représentations et modélisations de la société, constitue un enjeu transversal à l'ensemble des disciplines qui traitent de la culture matérielle.

Les archéologues ont démontré, par exemple, l'importance du sol dans le développement de la poterie, la domestication des plantes et des animaux et la formation des sociétés sédentaires (Stevanovic 1997; Wengrow 1998). Les anthropologues, quant à eux, ont commencé à examiner le rôle de la sélection des matériaux dans la construction des mondes sociaux (Ingold 2000; Damon 2004; Wagner 2007). De cet intérêt pour la transformation de la matière en forme, émerge une hypothèse, puissante mais déjà ancienne, à savoir que les idées abstraites sont formées par les techniques matérielles, hypothèse qui a fasciné les sciences sociales et historiques (Latour 1990; McLuhan 1962; Innis 1950). Toutefois, s'il est justifié de reconnaittre que l'intérêt pour le domaine matériel, trop négligé, a été très stimulant au plan théorique, on court le risque de produire des conclusions trop hâtives à propos de ce que la matière fait à l'esprit (Boivin 2008). L'attrait, pour cette façon de penser par les choses ${ }^{1}$, exercé sur des disciplines qui cherchent à échapper aux modèles dualistes, peut favoriser l'apparition d'une nouvelle forme de déterminisme évolutionniste dans laquelle «nous sommes ce que nous fabriquons ». On en perçoit les signes dans les disciplines universitaires tout autant que dans de nouvelles formes de localisme, vides de tout cadre de référence comparativiste (cf. Henare \& al. 2007; Knappett 2005).

Compte tenu de ces biais, notre article poursuit deux objectifs. Nous présenterons d'abord l'hypothèse suivante: ce n'est pas la matière en ellemême qui agit comme contrainte sur les processus techniques et l'imagination sociale, mais les manières de l'appréhender abstraitement selon une logique qui inspire les actions techniques de façon calculée. Nous pensons que cette logique est ancrée dans la nature relationnelle de l'action qui se découvre dans la matière (Gallese 2001). Nous pensons que ce potentiel 
de la matière est saisi par empathie, c'est-à-dire par une forme d'intersubjectivité. La nature de cette empathie avec la matière est cruciale pour expliquer le fait que les individus interprètent le monde social de façon abstraite, généralisable, souvent inchangeable. Nous examinerons ensuite la nature de cette empathie au travers de cas observés en Océanie. Le rôle de la matérialité dans l'imagination sociale a occupé une place prépondérante dans les études ethnographiques, cependant, curieusement et paradoxalement, les collections d'artefacts n'ont souvent été considérées et conservées qu'en tant que témoins périphériques de la description des mondes sociaux.

Alfred Gell (1993) dans ses travaux sur le tatouage en Polynésie est un des rares à avoir traité de la forme " artéfactuelle » des conceptions du monde social. À partir de riches sources archivistiques, l'auteur met en exergue la relation existant entre l'interprétation technique du tatouage et l'amplification relative de la distance sociale (spatiale) dans les institutions politiques. Pour Gell, le coefficient qui relie les interprétations techniques du tatouage en termes de blessures, marques et scarifications aux formes de différenciation sociale est issu d'une double conceptualisation de la peau comme frontière sociale et physique. Dans un ouvrage plus tardif, Gell (1998) essaie d'appliquer cette perspective aux traditions d'artefacts en affirmant de manière provocatrice que l'une des façons d'étendre ses capacités d'action consiste à donner une expression à son esprit dans des formes matérielles et techniques. Celles-ci sont, en conséquence, envisagées comme une forme de pensée intentionnelle et logique. Malade, il n’a pas pu établir une vision d'ensemble à partir de ces deux ouvrages mais nous en a laissé des indices dans un chapitre sur le style et la culture - qui semble être le moins lu et le moins apprécié de tous les chapitres de son ouvrage, désormais fondateur, Art and Agency $(1998-2009)^{2}$. Dans ce chapitre, il explore les compléments logiques et conceptuels de systèmes techniques en termes d'acte de mesure, de multiplication et de mise à l'échelle, qui inspirent la transformation de la matière en formes artéfactuelles. Décrite comme obéissant au «principe de la moindre différence », la "pensée-style » est perçue comme pouvant inclure à la fois la cosmologie et la création de variations dans les artefacts, et ce, d'une manière qui permet au « style » d'agir sur, plutôt que de suivre, la culture.

$\mathrm{Au}$ vu de la richesse des collections d'artefacts du Pacifique, nous posons comme hypothèse qu'il est possible d'étudier ce que nous qualifierons de « calcul $»^{3}$, concrétisé dans la fabrication d'artefacts, et qui implique des évaluations "non-mathématiques », concrètes et matérielles ${ }^{4}$, d'estimation (proportions et multiplications), en utilisant le modèle proposé par Gell pour saisir la distribution des pratiques du tatouage dans différentes institutions politiques en Polynésie. Notre article envisagera, sous un angle comparatiste, le calcul en tant qu'indicateur d'une empathie et d'un rapport intellectuel engagé et conscient avec la matière. Nous porterons notre attention sur la nature et la signification de cet engagement généralement peu étudié. 
L'énumération (l'inventaire ou le « comptage » des objets réels) en tant que logique technique (concrète) de la manipulation des matériaux émerge comme thème central de notre réflexion à propos du calcul dans les actions techniques. Cette notion de calcul nous ramène au texte classique d'Alleta Biersack (1982) sur le système de comptage Paielai qui implique le corps. Alleta Biersack propose d'envisager qu'il existe une logique mathématique alternative en Papouasie Nouvelle-Guinée et ce parallèlement à la science occidentale qui manipule les nombres de façon abstraite et indépendamment de tout motif (cf. Lévi-Strauss 1966). Largument en faveur d'une vision plus dualiste qu'évolutionniste ne fut pas entendu lorsque le texte de Biersack fut publié, et ce pour des raisons dépassant le cadre de cet article. Aujourd'hui, « la concrétude mal placée », que Biersack décrit comme caractéristique de la logique Paiela est à nouveau à la mode, alors que nous devenons nous-mêmes à nouveau conscients de la spécificité de la matière et de la technique, mais dans un monde matériel conçu en laboratoire et non plus issu du monde concret (Ball 1999 ; Küchler 2008).

Les parties et les touts, ainsi que leurs interrelations, ont été reconnus depuis longtemps comme des strates conceptuelles essentielles, à la base de la description fractale de la personne en Océanie (Strathern 1988 ; Wagner 1991). Ici, nous pousserons ces recherches plus loin en pistant les manières de traiter des parties et des touts dans le monde matériel et technique de la production d'artefacts, en tant que modélisations du corps social. Cette modélisation se réalise dans la production technique, au cours d'un processus de mise à l'échelle qui intègre la nature relationnelle de l'action. Les deux modalités d'énumération, évoquées plus haut, sont présentes en tant que stratégies d'intervention possible. Selon les contextes historiques l'une ou l'autre a pu être favorisée laissant dès lors son empreinte dans les collections d'artefacts. Ce sont précisément les classifications scientifiques occidentales imposées aux artefacts qui les ont réduits au rang d'indicateurs, voire de résidus, des relations sociales, plutôt que, comme le propose Biersack (1982), les résultats d'une logique alternative sophistiquée, orientée vers un processus de communication complexe fondé sur le calcul. Notre article tente de construire une façon différente d'étudier ces collections. Nous proposons de les envisager, non pas comme témoins de significations contextuelles ou oubliées depuis longtemps, mais bien comme témoins d'une forme « d'empathie avec les matériaux ». Cette empathie serait alors née de la découverte d'une logique relationnelle de l'action inscrite dans la matière et se serait maintenue dans les îles du Pacifique durant des siècles.

\section{La logique dans le faire}

Les malanggan constituent peut-être une des traditions d'artefacts les plus emblématiques d'Océanie. Il s'agit principalement de figures et de masques de bois aux formes entrelacées, de tailles différentes, qui jouent un rôle 
central dans les cérémonies funéraires et dont les motifs ajourés représentent des images dérivées autant des rêves que de l'écologie de l'île. Ce travail des figures ajourées, si caractéristique de la figuration des malanggan, est pratiqué dans le nord de cette grande île, située au nord-est de la Papouasie Nouvelle-Guinée (Küchler 2002) - connue à partir de la période coloniale sous le nom de Nouvelle-Irlande. D'après les documents d'archives, ce type de travail semble avoir connu une expansion lors des premiers contacts coloniaux, dans les années 1860, alors que de larges portions du territoire du nord de l'île étaient transformées en plantations de cocotiers tenues par des étrangers. Peu de temps après, une tradition de sculpture apparemment plus ancienne, connue sous le nom de Uli, produite dans le plateau de la Nouvelle-Irlande et distincte des malanggan du Nord par la figuration d'une silhouette trapue, à la surface sculptée dans un bois dur, allait disparaître. La tradition orale mentionne une tradition similaire dans le Nord, impliquant des rondins de bois dur pour former une plateforme de danse. Toutefois, personne n'a jamais expliqué de manière convaincante comment les malanggan en sont venus à remplacer les Uli, ou des pratiques analogues, en tant que techniques rituelles efficaces par excellence et dont les qualités sont aujourd'hui reconnues dans l'île tout entière. Nous avancerons dans cet article l'idée que la technique de sculpture de motifs ajourés à partir de bois tendre a donné son expression à une nouvelle manière d'organiser les relations avec le monde des morts, dont l'accès - source de régénération et de prospérité - avait été interrompu de manière soudaine et inattendue. Le bois tendre, plutôt que dur, est ainsi parvenu à représenter la promesse d'une action conçue de manière relationnelle. Le matériau sélectionné facilite la traduction technique d'une configuration prototypique, de nature conceptuelle et computationnelle. L'exécution technique de la sculpture sur bois a permis la multiplication de formes possibles, générées à partir d'une seule image prototypique. Cette multiplicité permettait alors de relier en une seule chaîne, les vivants et les morts dans une nouvelle forme de "politique de l'image ».

La période durant laquelle s'est développée la sculpture malanggan est marquée (si l'on se fie aux archives) par l'arrêt de la guerre. Cette dernière avait pris, dans le Nord, la forme d'une chasse aux têtes. Les âmes et les noms ainsi « capturés » représentaient un moyen d'augmenter le pouvoir ancestral (Taylor 1993). Nous nous en tiendrons ici à signaler que la fin de la chasse aux têtes marque la fin de la logique d'accumulation qui sous-tendait l'économie politique en Nouvelle-Irlande jusqu'au XIX ${ }^{e}$ siècle. Cette fin affaiblit et raréfia le pouvoir ancestral, dans la mesure où aucune substance ne pouvait venir augmenter et renforcer ce dernier. Afin de comprendre la raison 


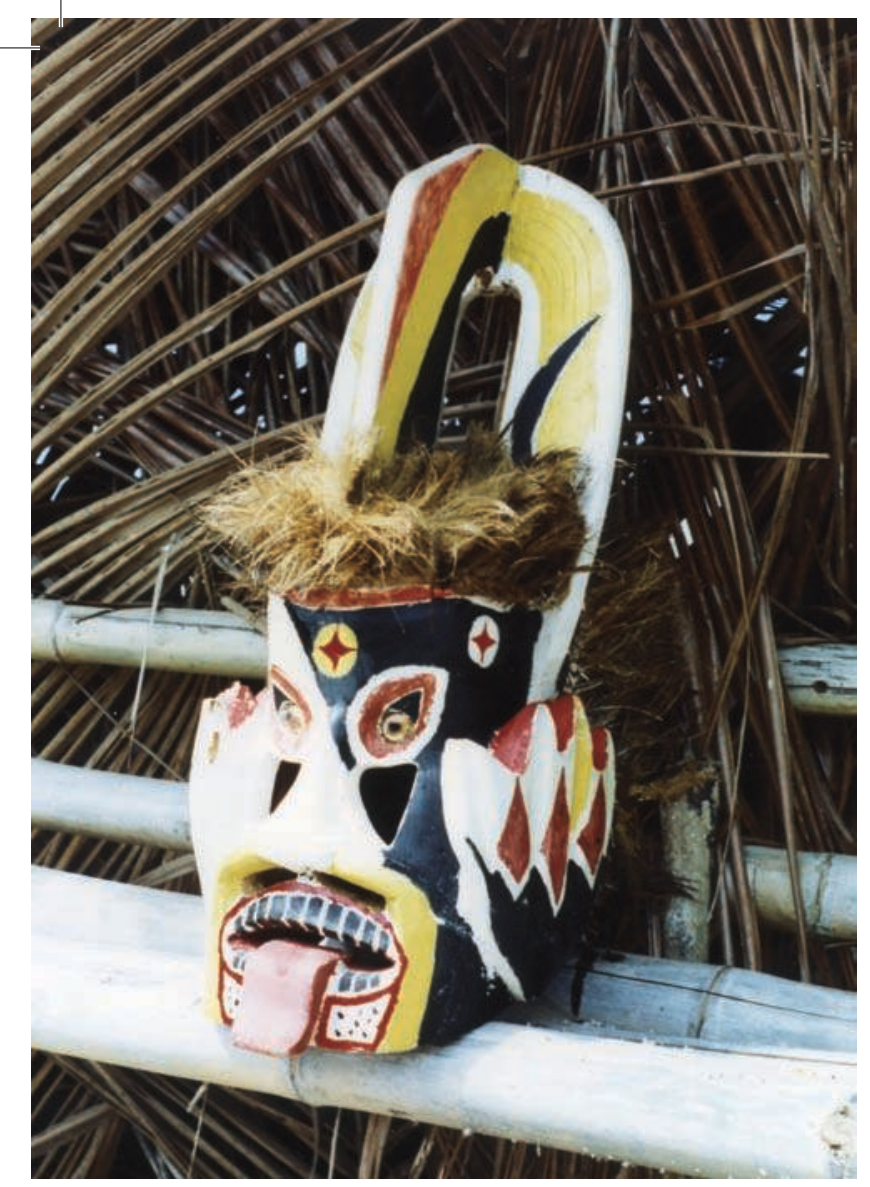

Masque malanggan

Les ajours dans les figures et les masques sont aujourd'hui moins importants. pour laquelle les motifs entrelacés devinrent centraux pour faire face conceptuellement au caractère désormais limité de la ressource, nous devons considérer brièvement les relations entre le visible et l'invisible, et plus particulièrement les artefacts tels qu'ils sont présents en tant que dons dans les échanges entre les vivants et les morts.

Pour les Neo-Irlandais d'alors et d'aujourd'hui, la communauté des vivants existe dans un monde parallèle à celui des morts. Étant conçus comme résidant sur une île au-delà de l'horizon appelée «Tinguen », les morts forment des collectivités connues sous le nom de fabung, que l'on peut traduire par « ceux qui sont faits pour être des ancêtres ». Il existe trois fabung nommés, qui peuvent être subdivisés en paires pour constituer six communautés, puis douze, puis vingt-quatre, et ainsi de suite. Les vivants sont identifiés à la communauté des morts de manière à ce que chaque personne appartienne à un fabung par sa mère. Ce n'est pas la naissance, toutefois, et encore moins la conception, qui définissent l'identité de la personne, mais l'acte de nommer, qui confère au nouveau-né un nom appartenant à un fabung. De tels noms de « mort » sont connus sous l'appellation collective de malanggan. Ils sont pensés conférer une ressemblance, qui lie les personnes vivantes avec celles qui peuplent le monde des morts. À la fin de sa vie, le nom d'une personne retourne dans le monde des morts au travers d'un «écran », le malanggan rejoignant un fabung. Les noms forment ainsi un cycle d'une génération à l'autre, et ne sont pas conçus comme augmentant en nombre. Bien que ceci semble simple en apparence, il en va autrement pour les gens vivant aujourd'hui sur l'île. Les complications surviennent lors de l'allocation de noms de mort à un fabung, étant donné que les écrans, qui servent de miroirs à double sens et de portail vers l'île des morts, peuvent attraper des noms qui appartiennent à des subdivisions « similaires » et pourtant distinctes de la collectivité des morts. Personne n'est vraiment sûr de savoir à quelle subdivision particulière de cette collectivité un nom est réellement censé appartenir. Ainsi, bien que le stock total de noms dérivés des morts soit considéré comme limité et inchangé, certaines communautés de morts sont pensées comme ayant plus de noms que d'autres, de la même manière que, durant la période de chasse aux têtes, certaines communautés de vivants accumulaient des noms aux dépens des autres.

La sculpture du bois tendre donne une expression concrète à cette image basée sur une logique d'accumulation, qui est localisée aujourd'hui fermement dans le monde d'au-delà de l'horizon. Les sculptures malanggan sont des écrans. La technique consiste à soustraire de la matière et les sculptures sont extrêmement incisées. La contre-forme est au moins aussi importante que la forme puisqu'elle est chargée d'« attraper » les noms qui la traversent pour rejoindre le monde invisible, où les morts sont censés résider. L'étendue des incisions des figures et des masques est en relation 
directe avec le nombre de noms de défunts qui doivent être transférés dans le monde des morts via la sculpture. Au début du xxe siècle, lorsque la plupart des figures très incisées et ajourées furent collectées, une seule sculpture était utilisée pour commémorer plusieurs défunts. Les morts devaient, selon la réglementation mise en place par l'administration coloniale, être enterrés dans de grands villages cimetières. Depuis l'indépendance en 1975, est réapparue la division des villages en moitiés complémentaires; chaque moitié étant segmentée en de nombreuses résidences, chacune ayant sa propre enceinte funéraire et son cycle de rituels funéraires culminant dans la production de figures malanggan exposées sur les tombes. De nos jours, il y a moins de noms enterrés et en attente d'être recyclés dans le monde des morts lorsque le décès d'un ancien provoque le début d'une nouvelle cérémonie malanggan. Par conséquent, les ajours dans les figures et les masques sont moins importants. Ces marqueurs de pouvoir découlant de l'accumulation des noms ancestraux, dans le monde de l'au-delà sont moins nombreux.

Toutefois, la logique qui consiste à compter les morts en les déduisant du nombre total de noms disponibles et en allouant à des sources de pouvoir discrètes n'a pas seulement servi de base à la technique de sculpture et aux formes sculpturales qui en résultent. Son effet le plus marquant et le plus durable se retrouve dans la technique conceptuelle qui génère des variations à partir de formes prototypiques nommées, correspondant elle-même aux petits groupes de noms ancestraux.

Ce qui est le plus remarquable au sujet des malanggan, en dehors de l'apparence creuse de leurs figures et de leurs masques, est leur échelle. Certaines sont énormes, trois mètres de haut, et nommées d'après les poutres des maisons (eikwar). D'autres sont de taille moyenne, de la taille d'un être humain, et sont nommées d'après les poteaux qui, installés verticalement sur l'avant et l'arrière d'une maison, soutiennent des murs de bambous (wagil). D'autres, enfin, sont petites et transportables, elles peuvent représenter un cabillaud géant ou un grand oiseau, un aigle par exemple. Toutes les figures ont un certain nombre de motifs correspondant à des groupes de pouvoir ancestral, inclus dans des noms totémiques malanggan (Küchler 2002). La forme qu'une figure doit prendre n'est pas «trouvée » lors du processus de prélèvement de matière, mais en amont, au cours d'une vision provoquée par l'absorption de décoctions de plantes.

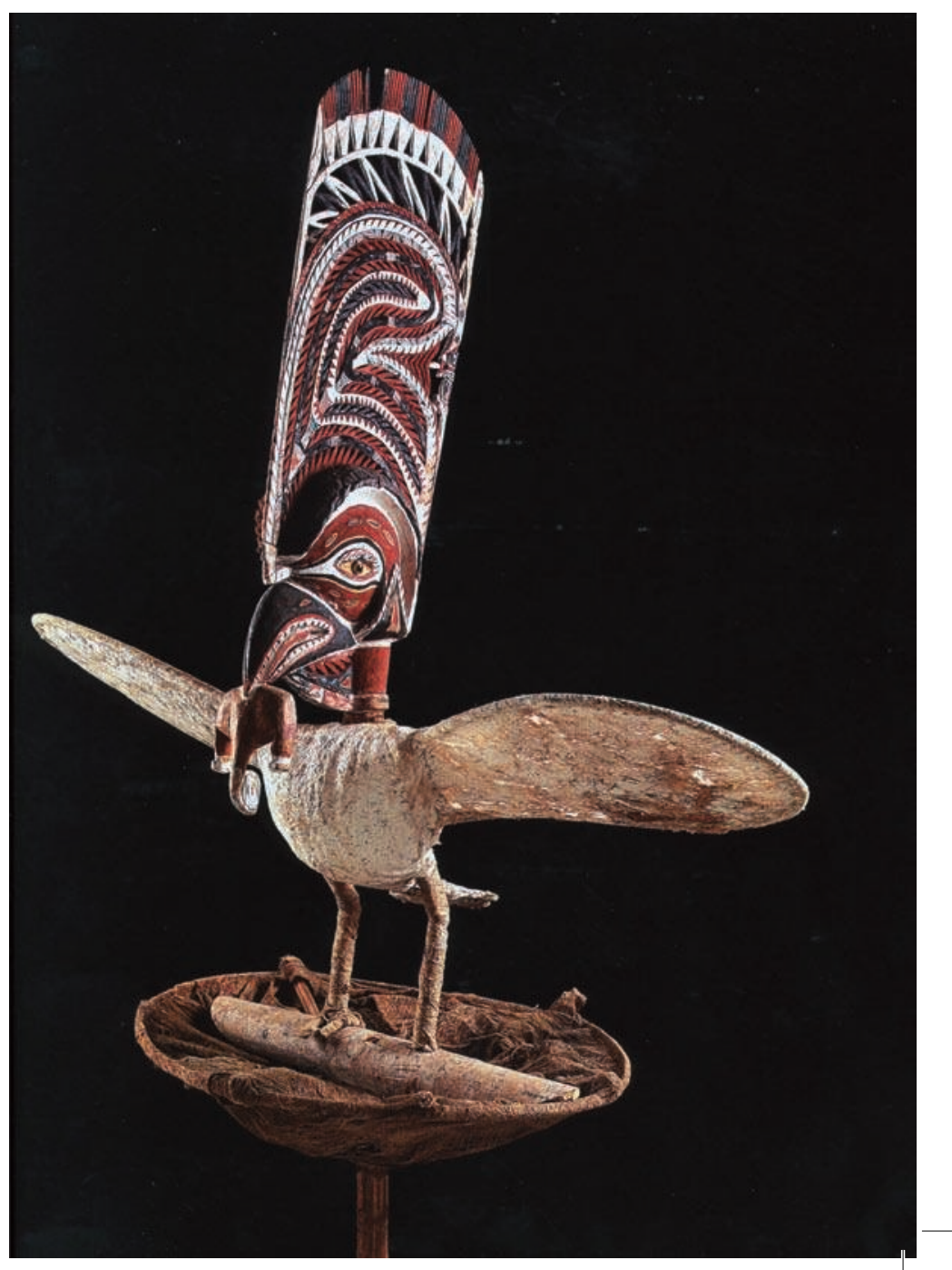

Oiseau malanggan

Écorces battues sur support en bambou.New Ireland. Chicago, Field Museum. 138876.1-3. 
Malanggan tressés en rond

Ils sont destinés à capturer les noms des femmes et des hommes non-initiés puis à les renvoyer vers les morts.

Warwara

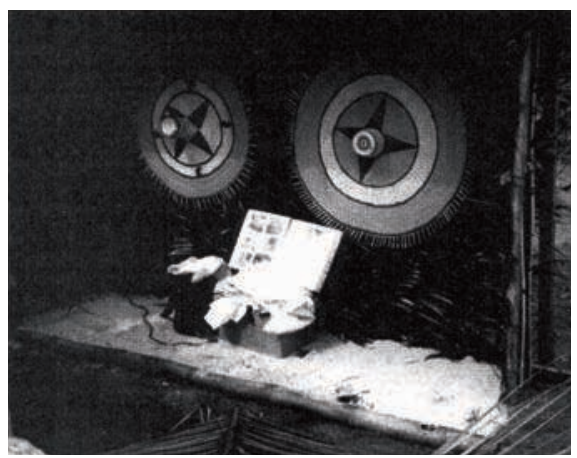

La soustraction, comme mode de calcul, impose des contraintes sur le type de matériau dans laquelle elle doit être réalisée. Plusieurs essences de bois sont accessibles sur l'île, mais une seule est considérée comme étant appropriée pour les malanggan. Il s'agit d'une essence que l'on trouve seulement dans les profondeurs de la forêt. Lorsque l'arbre adéquat est localisé, il est abattu puis découpé en tronçons. La taille des tronçons donne une indication quant à la complexité des intentions qui interviennent dans la fabrication de la sculpture. Laction technique de mise à l'échelle, qui possède pourtant une nature relationnelle, a été constamment ignorée dans les écrits anthropologiques, ceux-ci ayant plutôt accordé une importance analytique à la question des capacités représentationnelle des formes. Ici, nous voulons insister sur la signification

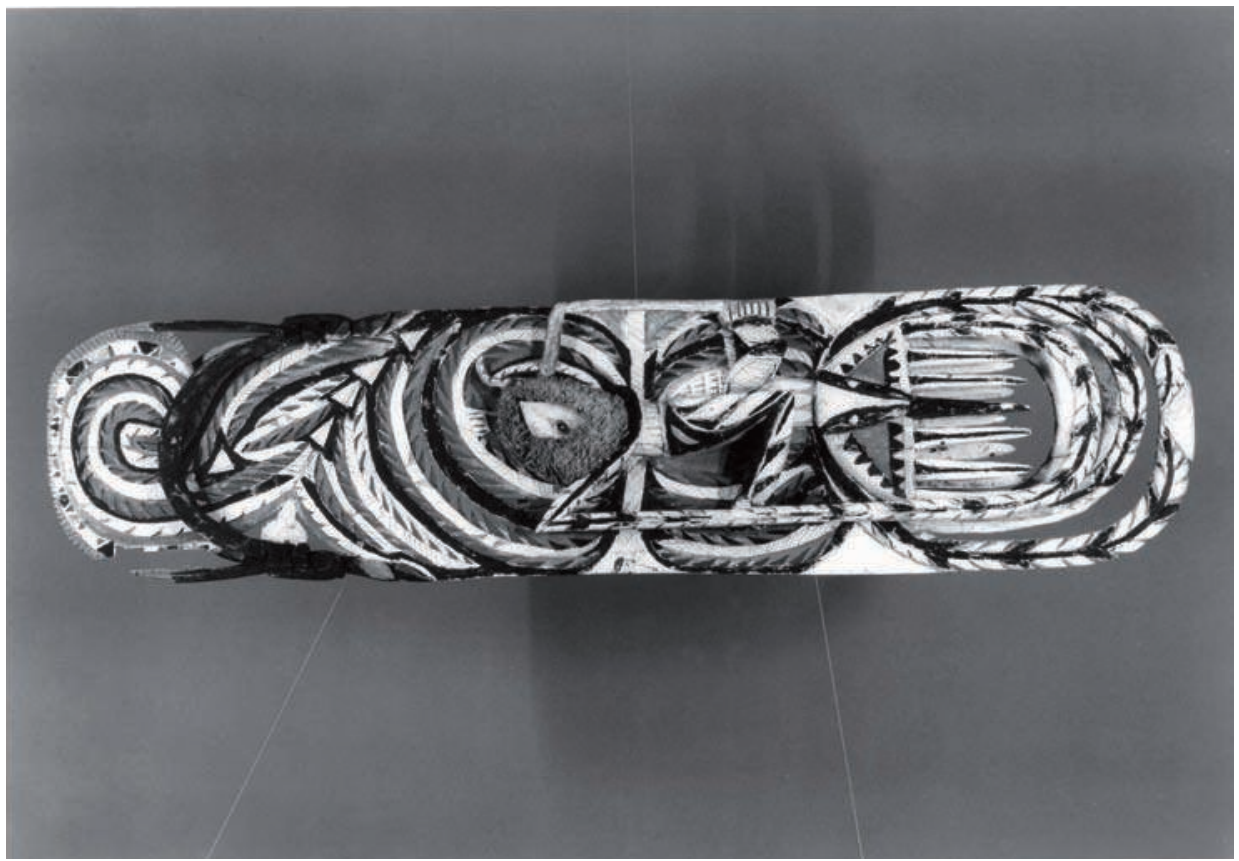


que possède cette mise à l'échelle, comme processus technique à mi-chemin entre le matériel et le conceptuel. Ce processus est pourvu d'une logique de l'énumération qui permet de transformer les quantités en énonciations appréciatives, relatives et relationnelles.

L'importance de la variation d'échelle dans les sculptures de NouvelleIrlande à l'époque actuelle, peut être évaluée à travers l'étude d'une technique de tressage circulaire qui consiste à créer des pièces de dimensions et d'échelle invariables. Cette technique se rencontre dans un type de malanggan qui a rarement attiré l'attention des chercheurs en raison de leur absence des collections. Ces malanggan tressés en rond sont de taille définie et fixe, d'une longueur calquée sur celle d'un avant-bras. Ils sont destinés à capturer les noms des femmes et des hommes non-initiés puis à les renvoyer vers les morts. Ces figures warwara capturent les noms de manière à les rendre socialement inactifs, c'est-à-dire de sorte qu'ils ne se rattachent à rien qui puisse en maintenir le souvenir. Ils sont brûlés, ne laissant aucune trace qui puisse avoir un effet quelconque sur les personnes concernées. Ils sont faits à partir de brindilles et de lianes, leur apparition occasionnelle dans les villages rappelle l'aspect sauvage et incontrôlé de la vie, associés aux esprits des personnes tuées à la guerre, aux pluies, au vent, aux bois et noix de coco échouées sur la plage et qui confirment la promesse d'une vie nouvelle dans l'île de l'au-delà. Chaque personne est censée avoir son propre warwara et c'est par le nom que celui-ci porte que l'on peut retracer sa propre descendance dans la lignée de sa mère. Par leur échelle indifférenciée, les warwara sont les indices de l'empathie entre les personnes et leur matrilignage, celui-ci étant alors conçu comme un groupe de personnes que l'on reconnaît intuitivement et chez qui l'on peut demeurer en sûreté, sans méfiance ni hésitation. Faits d'un fil continu qui se recoupe et qui maintient la construction, les warwara manifestent ainsi la nature auto réflexive et relationnelle des sentiers qui relient les clans matrilinéaires et sont utilisés pour traverser l'île.

\section{Repenser les collections ethnographiques}

Des « politiques de l'image » telles que celles ayant donné naissance aux malaggan ont laissé leur trace sur des milliers d'artefacts collectés et rassemblés dans nos musées. De telles économies politiques fondées sur des images génératrices sont à la source d'une forme d'« attraction cognitive », exercée par le caractère de vraisemblance et d'autosimilarité des formes.

Considérons à présent quelques objets de ces collections ethnographiques. À plusieurs milliers de kilomètres de la Nouvelle-Irlande, à Londres en Angleterre, dans un entrepôt de briques, parmi des milliers d'objets ethnographiques océaniens, languissent deux objets appartenant au British Museum. Au milieu d'une flotte de pirogues de tailles différentes, originaires des Îles Salomon, se dresse une grande pirogue composite, soutenue par un cadre d'aluminium. Juste à côté se trouve ce qui apparaît d'abord comme étant une pirogue monoxyle 
Détail du bol à nourriture en bois avec une tête de crocodile sculptée de Roviana,

trouvé dans les îles Solomon occidentales, British Museum. creusée, mais qui est en réalité un bol de nourriture. Celui-ci est décoré de motifs anthropomorphes. Ce qui peut être pris pour une proue est orné d'une large tête de crocodile. La pirogue composite et le bol ont été collectés en Nouvelle-Géorgie (province occidentale des Îles Salomon) il y a plus de cent ans. Le caractère impressionnant dans ces deux objets ne provient pas seulement de la qualité d'exécution investie dans leur production, mais aussi de leurs dimensions. En effet, la pirogue mesure plus de onze mètres de long, ce qui a pour effet de rendre toutes les autres pirogues minuscules. Le bol mesure plus de sept mètres de long. Il est souvent pris par les Occidentaux pour une pirogue monoxyle (Davenport 1997 : 318) - ce qui est facile à comprendre dans la mesure où les deux sont créés en creusant le bois d'un tronc. Cette impression est par ailleurs renforcée par sa localisation dans la réserve à côté des pirogues. Cette position suggère, d'une part, une parenté et confirme, d'autre part, que ce type de classification par volume fonctionne dans les réserves.

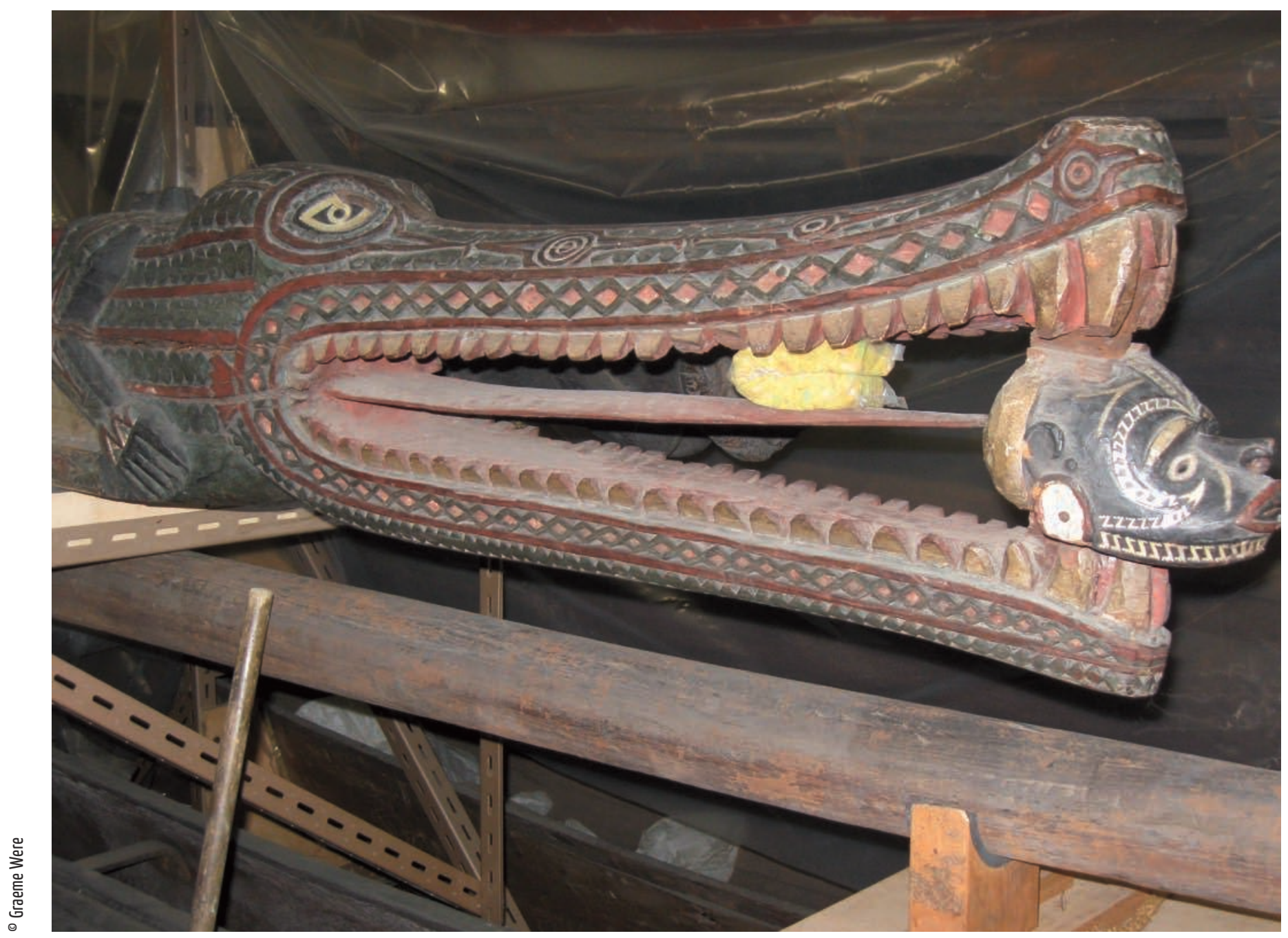


d'échelles opposées, ces techniques participent de manières différentes au modelage dynamique des relations sociales. Ceci met en lumière le fait que des matériaux spécifiques et les techniques qui leur sont appliquées comportent une capacité logique à indiquer les directions des actions futures. Sur cette base d'artefacts reliés par ces actions techniques d'addition et de soustraction, nous pouvons parcourir les contextes ethnographiques de production de la pirogue et du bol, afin d'explorer la manière dont les modalités d'énumération rendent compte de la nature relationnelle de l'action, et ceci à travers le processus de mise à l'échelle.

La grande pirogue de Nouvelle-Géorgie, connue localement sous le nom de tomako, était utilisée lors d'expéditions à la fois de chasse aux têtes et de capture d'esclaves dans les îles voisines. Les pirogues, parfois groupées, emportant entre vingt et trente guerriers, attaquaient les populations côtières des autres îles, de sorte que de nombreuses populations se déplacèrent pour s'installer à l'intérieur des terres. L'administrateur colonial, C. M. Woodford en poste aux Îles Salomon à la fin du xixe siècle, rapporte de sa visite comment quelque quarante têtes furent rapportées durant son séjour de deux semaines en Nouvelle-Géorgie en 1886 (1909: 510). Cette pratique se poursuivit jusqu’à la pacification par la loi coloniale britannique. Woodford aborde la question de l'échelle dans le récit qu'il fait à propos de sa tentative de rapporter pour le British Museum une pirogue de guerre. Cette dernière mesurait plus de treize mètres et avait été prise par les Britanniques. Woodford n'ayant pas réussi à réunir les fonds nécessaires pour son transport, la pirogue fut finalement acquise par un musée allemand. Woodford poursuit tout de même son récit en rapportant comment, quelques années plus tard, il a finalement fait l'acquisition d'une pirogue plus petite. Il s'agissait d'une « copie fidèle » de la première pirogue. Ce modèle de près de 13,4 mètres fut déposé au Bethnal Green Museum (Woodford 1909 : 511). Il demeure toutefois introuvable actuellement.

Les notes ethnographiques de Woodford décrivent comment la pirogue de guerre fut construite à partir d'un assemblage de matériaux remplissant à la fois un rôle fonctionnel, pour la navigation, mais aussi un rôle symbolique. Edvard Hviding (n.d. : 17) décrit également comment les proues et les poupes imposantes des pirogues furent construites à partir d'un type particulier de bois léger connu sous le nom de tangovo. De très haute taille, tangovo peut être aperçu dépassant de la canopée lorsque l'on est en mer. Hviding poursuit en indiquant que les défunts étaient souvent placés à la base de cet arbre, maintenus en position assise par les racines émergentes. L'esprit du défunt était alors censé remonter le long du tronc vers la canopée, puis traverser la mer vers l'ouest, en direction du domaine ancestral situé du côté du couchant. Ainsi les qualités de légèreté et de solidité du bois de tangovo étaient utilisées non seulement pour améliorer la tenue en mer et la vitesse de la pirogue, mais elles correspondaient également à une « logique matérielle » par son association aux pouvoirs spirituels et aux ancêtres.

Tandis que la pirogue monoxyle est fabriquée par la soustraction de la matière à partir d'un seul tronc, Woodford observe comment la pirogue de guerre de Nouvelle-Géorgie mobilise une technique additive. Les planches 
sont ligaturées aux traverses par l'intermédiaire de fibres passées dans des trous (Woodford 1909 : 508-509). Les traverses sont mises en forme et attachées fermement ensemble avant d'être mises à sécher dans la maison des pirogues jusqu'à leur utilisation lors de l'assemblage. Lorsque la coque est complète, une couche épaisse de résine noire de parinarium est appliquée aux jointures pour rendre l'embarcation étanche. L'inspecteur naval H. B. Somerville donne davantage de détails sur la fabrication (Somerville 1897). Il décrit la façon dont les planches sont amincies jusqu'à ce que leur épaisseur soit inférieure à $1 \mathrm{~cm}$, parfois moins, tout en laissant une nervure sur la longueur de la planche afin de la consolider. Les planches sont pliées en force entre deux poteaux plantés dans le sol. L'ensemble de la pirogue est cousu d'un cordage à trois brins en fibre de coco, avant qu'une nouvelle couche de résine de parinarium ne soit appliquée pour l'étanchéification. Somerville ajoute que la pirogue doit être gardée à l'abri de la pluie durant une période allant d'une semaine à dix jours, pour permettre au mastic de sécher. Des traverses sont ensuite construites à l'intérieur de la pirogue pour maintenir sa forme initiale. Somerville précise qu'en dépit de l'absence de pont, "on trouve une sorte de plateforme de rondins, similaire à celles que l'on trouve dans certaines maisons et sur lesquelles on plaçait les têtes des victimes, ou bien qu'on utilisait pour transporter la nourriture ou autre chose; et on trouve également des sortes de crochets à intervalles réguliers sur la longueur du bateau, pour transporter les lances, les canes à pêche, etc. » (Somerville 1897 : 370). À l'inverse de la pirogue monoxyle plus lourde, l'embarcation composite plus légère et plus stable permet des voyages importants. Sa taille et sa vitesse facilitent également le déploiement d'un formidable effectif militaire.

Comme l'ont souligné Haddon et Hornell (1936) pour les autres pirogues des Îles Salomon, la coque des embarcations de Nouvelle-Géorgie présente une variété de motifs décoratifs. Comme pour les proues des pirogues de la kula (Campbell 2002), l'application de traits décoratifs a une finalité magique. Elle est censée réduire les risques et, par conséquent, d' augmenter les chances de réussite des expéditions maritimes. La surface extérieure de la coque, en particulier autour de la proue et de la poupe, est couverte de motifs représentant des animaux et des oiseaux, utilisant des incrustations de nacre d'huître perlière, ce qui permet de faire ressortir la figure sur le fond noir. Les motifs sont supposés jouer un rôle protecteur contre les esprits marins malveillants (Hviding n.d. : 18). À la fois par leur style et leur technique, ces motifs ressemblent à ceux que l'on retrouve sur un certain nombre d'autres supports, incluant les tatouages faciaux et les sculptures, présents également dans d'autres îles.

Les expéditions de chasse aux têtes et les déplacements de guerriers de Nouvelle-Géorgie qu'elles occasionnaient, occupaient une place importante dans une économie politique centrée sur le mana, un pouvoir invisible et une force vitale capables de régénérer l'environnement social et politique. En Nouvelle-Géorgie, le mana est concentré dans le crâne des chefs défunts et de leurs proches, utilisés comme sources de pouvoir spirituel. Ceci était 
aussi valable pour les têtes et les crânes d'étrangers pris lors des guerres et qui étaient réintroduits dans les pratiques religieuses locales (Hviding n.d. : 6). Durant ces expéditions, les guerriers espéraient revenir non seulement avec des têtes, mais aussi avec des captifs, qui étaient alors réduits en esclavage et employés à la production de richesses constituées de coquillages, tels que les anneaux en bénitiers (Hviding n.d. : 7). La prise des têtes permettait de rapporter du mana venu de l'extérieur et de diminuer celui des ennemis, affaiblissant leur économie spirituelle et leur volonté politique, en les laissant être harcelés par les fantômes des morts qui ne pouvaient plus être enterrés sur leur terre ancestrale. La préparation et l'organisation de ces expéditions servaient ainsi à nourrir une économie rituelle florissante dans laquelle la manipulation comme la perturbation le mana pouvait assurer le succès de l'exploitation des ressources terrestres et maritimes.

On voit alors plus clairement comment les efforts investis dans la sélection des matériaux et dans l'exécution technique de la construction des pirogues pouvaient devenir un instrument dans cette économie politique centrée autour du mana, ainsi qu'à la reproduction des relations sociales dans la région. Nous pourrions dire que l'échelle de la pirogue devient un indice du pouvoir exercé par les clans organisateurs, d'abord par leur capacité à construire de grandes pirogues mais aussi, et de manière plus importante, par leur capacité à préparer des attaques contre leurs rivaux. Ceci leur permettait d'imaginer un système expansif de relations sociales avec l'extérieur par l'entremise de la guerre et de l'accumulation de mana (cf. Harrison 1993). La préparation, l'orchestration et le déploiement des ressources naturelles et des troupes de guerriers, faisaient ainsi intégralement partie de cette forme calculée de construction de pirogues composites - qui à leur tour étaient destinées à la création de relations sociales externes. Ainsi, les pirogues de grande échelle pouvaient être déployées sur de vastes territoires et, de cette manière, réintégrer du mana afin de régénérer les forces vitales locales.

Tandis que la première moitié de cette analyse s'est concentrée sur l'augmentation d'échelle par l'addition de planches sur les coques des pirogues de Nouvelle-Géorgie (nous pourrions étendre ce principe à d'autres pirogues composites des Salomon), les techniques soustractives associées aux bols des Îles Salomon présentent une série de similitudes mais aussi de contrastes. Comme dans le cas des pirogues composites, les collections d'artefacts ethnographiques des musées fournissent des preuves matérielles de l'importance des bols en bois sculpté produits dans tout l'archipel, dont de nombreux exemples présentent ces incrustations complexes de coquilles d'huîtres perlières, ainsi que des motifs d'oiseaux et d'animaux. Non seulement ces traits décoratifs incrustés ressemblent à ceux des proues et des poupes des pirogues, mais il est également remarquable de voir combien le bol ressemble à une version réduite de la pirogue composite. Il semble en effet que les bols aient été fabriqués aux mêmes endroits que les pirogues.

Ces bols de bois sculptés étaient utilisés pour servir la nourriture, notamment un mélange de taro et d'amandes, pilé jusqu'à l'obtention d'une sorte de porridge. Comme l'a montré Davenport, il existe de nombreuses 
classes de bols dans la partie orientale des Îles Salomon, certains étant conservés dans la maison des pirogues où les repas rituels avaient lieu. D'aucuns étaient associés aux esprits des morts, notamment à ceux morts violemment, tandis que d'autres étaient utilisés à des fins plus prosaïques.

Bol en bois - Îles Salomon UCL Ethnography Collection, R.0014.

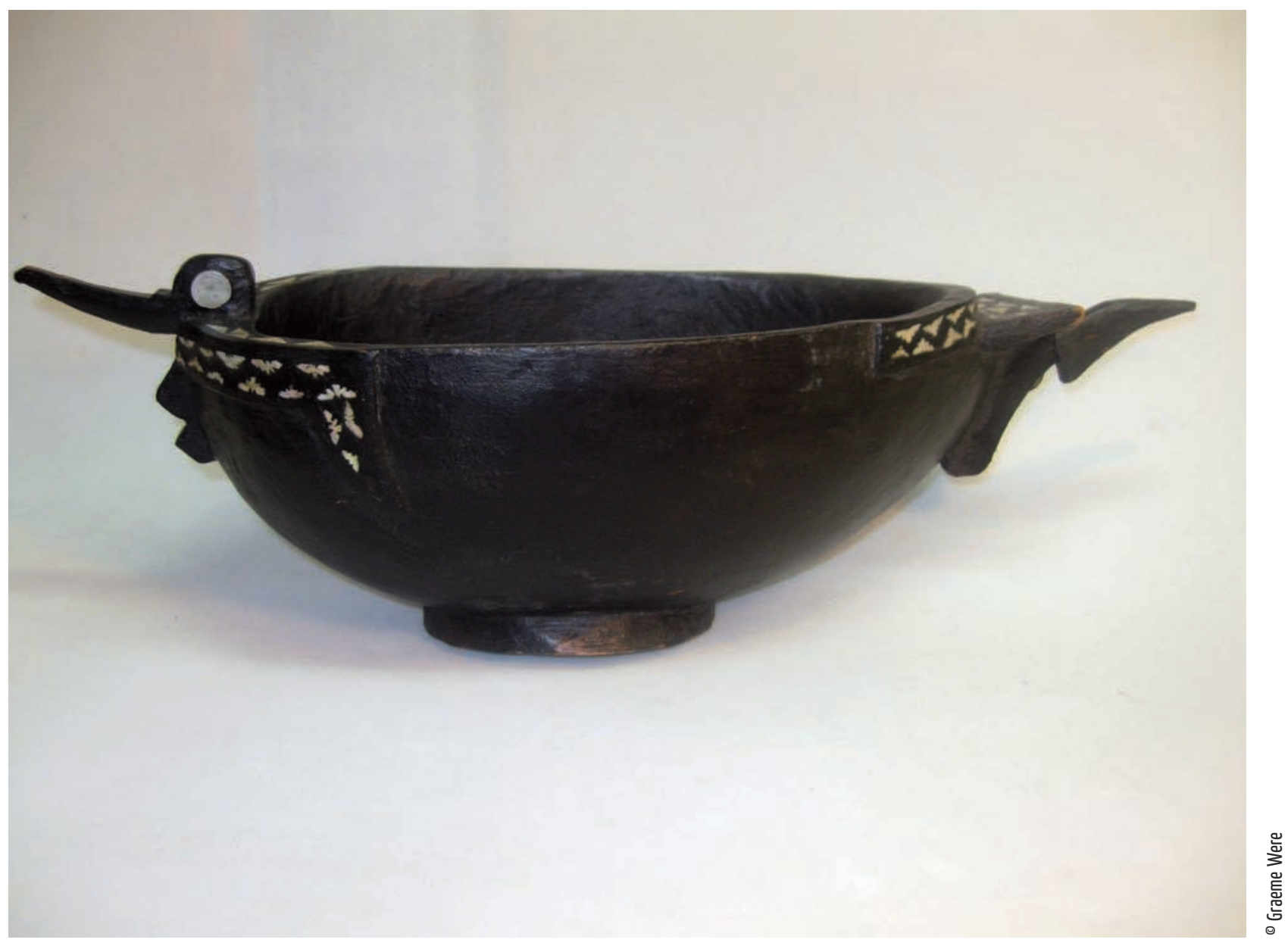

Les bols disposant d'un pied, comme l'indique Davenport, sont associés aux esprits tutélaires. La quantité de nourriture qu'ils peuvent contenir dépend du nombre d'hommes censés s'y nourrir (Davenport 1997 : 317). Certains bols mesurant entre 10 et 50 centimètres, d'autres, de plus d'un mètre de long, sont dédiés à des groupes plus nombreux. Selon Davenport, un bol peut être utilisé par un homme âgé puissant et par les hommes plus jeunes d'une même maisonnée (Davenport 1997 : 317). Les bols peuvent être rangés près de l'ouverture des maisons, sous les chevrons, ce qui permet à la fumée des foyers de circuler, invitant alors les esprits à rendre visite au bol (Davenport 320). Avant d'être utilisé, il est lavé dans la mer, puis rapporté dans la maison des pirogues où la fête doit avoir lieu. Davenport décrit comment la 
Pirogue dans les hangars Vella Lavella, Îles Solomon. Argentique de la fin du xixe siècle. Oc1927,1022.1.

Autorisation gracieuse de reproduction de la part des administrateurs du British Museum. nourriture, composée d'une sorte de pâte spéciale, faite d'igname ou de taro cuit et parfois accompagnée de poisson ou de porc, peut être d'abord offerte aux esprits avant d'être consommée par les humains.

Davenport a aussi documenté la manière dont l'un des bols, de près d'un mètre de long, était utilisé durant les initiations. D'autres types de récipients d'une longueur de 1 à 4 mètres sont connus sous le terme de « grands bols» (Davenport 1997 : 318). Certains peuvent être utilisés une seule fois durant une cérémonie d'échange avec des partenaires d'autres communautés. De tels bols, bien que peu décrits, pourraient renvoyer à celui mentionné plus haut, conservé dans les collections mélanésiennes du British Museum et décrit par Edge-Partington (1903) comme étant une « auge à nourriture de Rubiana, Nouvelle-Géorgie ». Davenport rapporte également comment ces bols pouvaient être tirés jusqu'à la mer par les enfants, qui les utilisaient comme embarcations dans leurs jeux.

Davenport fait allusion à la relation complémentaire entre le bol et la pirogue lorsqu'il contextualise l'utilisation rituelle du bol. Pendant la saison de la pêche à la bonite, la prise pouvait être cuite dans la maison des pirogues et être consommée dans ces bols, accompagnée d’une préparation à base de pâte spéciale (Davenport 1981). D’autres repas rituels avaient lieu dans cette maison des pirogues, en particulier ceux accompagnant les initiations masculines, qui impliquaient également la préparation de pudding dans les récipients en bois. La classe et le type de bol paraissent ainsi conditionner le type d'activité organisée et le nombre d'hommes qui y mangent.

Comme la pirogue, le bol à pudding est fait d'un bloc monoxyle de bois tendre, issu du tronc d'un arbre particulier (voir Davenport 1997 : 322). De la même manière que pour les malanggan, la forme définitive du bol

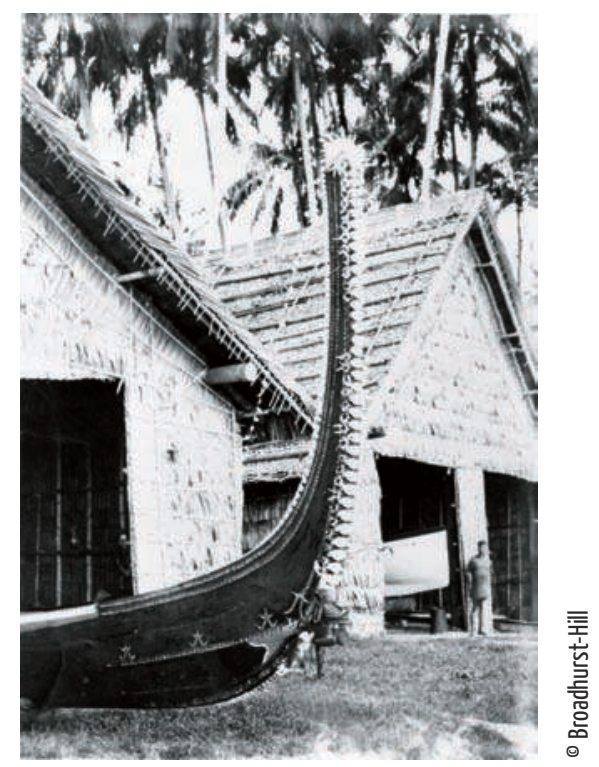


et les types d'animaux représentés se révèlent lors de rêves ou de visions communiqués par les esprits tutélaires avant que le sculpteur ne commence son travail (Davenport 1997 : 319). Durant le processus de fabrication, le sculpteur découpe le bois en une série de rectangles dont les proportions sont associées par un système d'échelle relative (1997 : 322). Davenport note que « tous les détails émergent graduellement des masses rectangulaires intermédiaires » (1997 : 322). Ainsi, quatre rectangles vont émerger à des échelles variables: un grand rectangle à partir duquel le bol sera sculpté; deux rectangles plus petits pour les poignées; et un dernier pour la base du socle. Comme le processus technique qui combine les niveaux conceptuel et matériel, le processus de mise à l'échelle évoque la logique qui sous-tend l'énumération, au travers de l'articulation qui transforme des quantités en expressions évaluatrices, relatives et relationnelles des activités rituelles.

\section{$\mathcal{E}$}

Notre analyse de la technique montre comment la pirogue et le bol jouent sur des économies d'échelle contrastées mais complémentaires. La pirogue, par son jeu de ligatures des planches entre elles, met l'accent sur un système d'accroissement d'échelle, indice de l'empathie qui gouverne la manière dont les personnes pensent les relations sociales qui ne peuvent être réalisées qu'au travers du voyage. C'est l'action de prendre et de rapporter sur la terre ancestrale des têtes et des captifs qui a un rôle instrumental dans les rites de succession au sein de la société de Nouvelle-Géorgie et dans cette économie du mana qui augmente les possibilités d'exploitation de la terre et des ressources. Le bol, quant à lui, joue un rôle complémentaire, alors que les têtes et les captifs nourrissent le mana à l'intérieur, le bol joue sur la notion de nutrition et de régénération de ceux qui sont à l'extérieur. Comme Davenport l'affirme dans son ethnographie des Îles Salomon orientales, le remplissage du bol par le pudding ne concerne pas seulement l'acte de nourrir les membres de sa famille et de la communauté, mais aussi celui de donner de la nourriture aux ancêtres, les esprits tutélaires qui inspirent la construction des bols (Davenport 1981, 1997). La prospérité du bol repose sur ce qui est à la fois « déjà connu », imaginé et réalisable dans la gestion des relations avec les vivants et avec les morts. L'opération de mise à l'échelle est rendue explicite par la taille du bol : plus il est grand, plus le statut de l'ancêtre pour lequel le récipient est fait est important. Mais, en même temps, on découvre que plus le bol est grand, plus le nombre d'hommes qui peuvent s'y nourrir est important. Cette double conséquence renvoie à une notion d'inclusion qui contraste avec celle portée par la pirogue. Tandis que dans le cas de la pirogue, il s'agit d'inclure des relations externes qui restent à réaliser, le bol soutient des relations sociales qui préexistent déjà à travers les réseaux d'échanges. 
La pirogue comme le bol des Îles Salomon, mais aussi les malaggan du Nord de la Nouvelle-Irlande, montrent comment les techniques d'énumération ont un rôle central dans la sélection et la manipulation technique des matériaux. Le calcul en jeu dans les processus de fabrication et de pensée, via les artefacts, sous-tend la nature dispersée et relationnelle du monde matériel et technique en Océanie. Ce monde apparaît comme un système qui articule des parties et des touts. Au vu de cette séquence logique d'actions, fondées sur la possibilité que présentent les matériaux de répondre à certaines attentes et de se prêter à des actions spécifiques, on commence à percevoir combien il est important d'attirer l'attention sur cette « empathie » avec les matériaux (Gallese 2001). Cette empathie correspond ainsi à une appréhension hautement abstraite et conceptuelle du monde social concret.

La « concrétude » mise en avant par Aletta Biersack dans son analyse des techniques corporelles de comptage n'attend plus que d'être exhumée des réserves poussiéreuses des musées ethnographiques et hissée vers les domaines théoriques de l'anthropologie.

(Traduit de l'anglais par Ludovic Coupaye) 


\section{NOTES}

Photo d'ouverture : Découpage Malanggan, Maru, New Ireland.

1. « Thinking through things » est le titre d'un ouvrage paru en 2007, dirigé par Henare \& al., qui propose une révision des approches classiques des objets. L'expression «thinking through », «penser au travers de » signifie à la fois à « l'aide de » et « repenser de manière à corriger $»$. Dans son acception anthropologique anglo-américaine, l'expression est commentée dans l'ouvrage de Shweder, Thinking through cultures, 1991, qui montre comment elle s'inscrit dans un vocabulaire issu des écoles deconstructionnistes.
En soi, « thinking through things », peut se traduire par « repenser les choses » $[\mathrm{NdT}]$.

2. Traduit en français par Sophie et Olivier Renaut en 2008: sous le titre L'art et ses agents, une théorie anthropologique. 2009 Dijon, Les Presses du Réel [NdT].

3. Le terme « calculus » en anglais regroupe le calcul différentiel et intégral [NdT]

4. Eglash (1999) et Ashler (2002) ont avancé l'idée que, bien que des idées mathématiques complexes puissent être déployées en pratique dans des sociétés non occidentales, ces idées ne sont pas nécessairement classées.

\section{RÉFÉRENCES}

Ascher, Marcia, 2002, Mathematics Elsewhere: An Exploration of Ideas across Cultures Princeton. N.J., Oxford, Princeton University Press.

Ball, Phillip, 1999, Made to Measure: New Materials for the 21 st Century Princeton. N.J., Chichester, Princeton University Press.

Biersack, Aletta, 1982, «The Logic of Misplaced Concreteness: Paiela Body Counting and the Nature of the Primitive Mind », American Anthropologist 84 (4) : 811-29.

Boivin, Nicole, 2008, Material Cultures, Material Minds: the Impact of Things on Human Thought, Society and Evolution Cambridge. Cambridge University Press.

Campbell, Shirley F., 2002, The Art of Kula. Oxford, Berg.

Damon, Frederick H., 2004, «On the Ideas of a Boat: from Forest Patches to Cybernetic Structures in the Outrigger Sailing Craft of the Eastern Kula Ring, Papua New Guinea ». in Beyond the Horizon: essays on myth, history, travel and society eds., C. Sather and T. Kaartinen. Finnish Literature Society, Helsinki :12344.

Davenport, William H., 1981, «Male Initiation in Aoriki: Man and the Spirits in the Eastern Solomon Islands », Expedition 23 (2): 4-19.

Davenport, William H., 1997, « Ritual Bowls of the Eastern Solomon Islands », Baessler-Archiv, Neue Folge 45: 315-331.

Edge-Partington, J., 1903, « Food Trough from Rubiana, New Georgia », Man 3: 161-62.

Eglash, Ron, 1999, African Fractals: Modern Computing and Indigenous Design New Brunswick. N.J., Rutgers University Press.

Gallese, Vittorio, 2001, The «Shared Manifold Hypothesis: from Mirror Neurons to Empathy », Journal of Consciousness Studies 8 (5-7): 33-50.

Gell, Alfred, 1993, Wrapping in Images: Tattooing in Polynesia. Oxford, Clarendon Press.

Gell, Alfred, 1998, Art and Agency: an Anthropological Theory. Oxford, Oxford University Press Traduit en français par Sophie \& Olivier Renaut en 2009: sous le titre L'Art et ses agents, une théorie anthropologique. 2009. Dijon, Les Presses du Réel

Haddon, A. C. and J. Hornell 1936, «Canoes of Oceania (Special Publication No. 27) Honolulu, Hawaii », Bishop Museum Press. 
Harrison, Simon, 1993, The Mask of War: Violence, Ritual and the Self in Melanesia. Manchester, Manchester University Press.

Henare, A., M. Holbraad and S. Wastell eds., 2007, Thinking through Things : Theorising Artefacts. Ethnographically Abingdon, Routledge.

Hornell, James, 1939, « Origins of Plank-Built Boats », Antiquity 13 (49) : 35-44.

Hviding, Edvard, n.d. « War Canoes of New Georgia », Unpublished Manuscript.

Ingold, Tim, 2000, The Perception of the Environment: Essays on Livelihood, Dwelling and Skill. Londres, Routledge.

Innis, Harold A. 1950, Empire and Communications. Toronto, University of Toronto Press.

Knappett, Carl, 2005, Thinking through Material Culture: an Interdisciplinary Perspective. Philadelphia University of Pennsylvania Press.

Küchler, Susanne, 2002, Malanggan: Art, Memory and Sacrifice. Oxford, Berg.

Küchler, Susanne, 2008, «Technological Materiality: Beyond the Dualist Paradigm ». Theory, Culture and Society. Vol 25 (1): 101-120.

Latour, Bruno, 1990, «Drawing things together ». in Lynch, M. \& Woolgar, S. eds., Representation in Scientific Practice. Cambridge, MA, The MIT Press : 19-68.

Latour, Bruno, 1996, Aramis, or, the Love of Technology. Cambridge, MA; Londres, Harvard University Press.

Law, John 1992, " Notes on the Theory of the Actor-network: Ordering, Strategy, and Heterogeneity », Systems Practice 5 (4): 379-93.

Lemonnier, Pierre, ed., 1993, Technological Choices: Transformation in material culture since the Neolithic. Londres et New York, Routledge.

Levi-Strauss, Claude, 1966, The Savage Mind. Londres, Weidenfeld and Nicholson.

McLuhan, Marshall, 1962, The Gutenberg Galaxy: the Making of Typographic Man. Londres, Routledge.

Sigaut, François, 2002, « Technology » in Tim Ingold ed., Companion Encyclopedia of Anthropology. . Londres, Routledge: $420-59$

Somerville, Boyle T., 1897, «Ethnographical Notes in New Georgia, Solomon Islands », Journal of the Royal Anthropological Institute, 26: 357- 412.

Stevanovic, Mirjana, 1997, «The Age of Clay: The Social Dynamics of House Destruction », Journal of Anthropological Archaeology, 16 (4): 334-95.

Strathern, Marilyn, 1988, The Gender of the Gift: Problems with Women and Problems with Society in Melanesia. Berkeley, CA, Londres, University of California Press.

Strathern, Marilyn, 1991, Partial Connections, Savage. MD, Rowman et Littlefield.

Taylor, Anne Christine, 1993, «Remembering to Forget: Identity, Mourning and Memory Among the Jivaro », Man N.S, 28 (4) : 653-78.

Wagner, Roy, 1991, «The Fractal Person ». in M. Godelier \& M. Strathern eds., Big Men and Great men: Personifications of Power in Melanesia. Cambridge, Cambridge University Press : 159-73.

Wagner, Monika, 2007, « Hans Haacke, Searth Samplings for the Bundestag: Materials as Signs of Political Unity », Journal of Material Culture, 12 (2) : 115-30.

Wengrow, David, 1998, «The Changing Face of Clay: Continuity and Change in the Transition from Village to Urban Life in the Near East », Antiquity, 72: 783-95.

Woodford, C. M, 1909, «The Canoes of the British Solomon Islands », Journal of the Royal Anthropological Institute, 39: 506-16. 


\section{RÉSUMÉ}

Empathie avec la matière: comment repenser la nature de l'action technique.L'importance des matériaux est souvent minimisée dans l'analyse des systèmes sociaux et techniques alors qu'ils façonnent les bases mêmes du monde dans lequel nous vivons. Cet article se propose de questionner cette omission en attirant l'attention sur la nature des matériaux et des actions calculées qu'ils occasionnent. Nous soutenons que le potentiel transformatif des matériaux joue un rôle essentiel quant à leur choix et leur utilisation, lequel potentiel va de pair avec une certaine empathie, une « intersubjectivité », dont la nature est déterminante pour comprendre comment les individus interprètent leur monde social de manière abstraite, généralisable et souvent immuable. Nous examinons la nature de l'empathie avec les matériaux à travers une série d'études de cas ramenés du Pacifique, où le rôle du concret dans l'imagination sociale occupe une place importante dans les études ethnographiques, contrairement aux collections d'artefacts qui, curieusement, demeurent en marge, un simple témoignage de la description du monde social.

\section{ABSTRACT}

Empathy with the matter: how to reconsider the nature of the technical action. Materials are often downplayed in the analysis of social and technical systems and yet materials shape the very basis of the world we live in. This paper sets out to challenge this oversight by drawing attention to the nature of materials and the acts of calculation they engender. The transformative potential of materials, we argue, plays a fundamental role in their selection and uptake and is met with an empathy that is shared intersubjectively, the nature of empathy with materials being crucial for explaining how individuals interpret their social world in abstract, generalizable and often unchanging ways. We examine the nature of empathy with materials through a series of case studies taken from the Pacific, where the role of the concrete in social imagination has figured prominently in ethnographic studies, yet where artefact collections have curiously remained bystanders to the depiction of social worlds.

\section{MOTS CLÉS}

Matériaux, empathie, logique, calcul, collections océaniennes.

\section{KEYWORDS}

Materials, empathy, logic, calculation, Oceanic collections. 